\title{
Mechanics of side-slipping in alpine skiing. Braking and skidded traversing
}

\author{
Serguei S. Komissarov ${ }^{1} \mathbb{D}$
}

Accepted: 31 August 2021 / Published online: 14 September 2021

(c) The Author(s) 2021

\begin{abstract}
A recently proposed simple approximate theory of snow machining is applied to modelling of several basic manoeuvres of alpine skiing: fall-line side-slipping, traversing, and hockey stop. The results agree with the skiing practice and explain the abnormally high friction reported in previous field studies. They also prepare foundation for future rigorous testing of the theory, which will determine its accuracy and limits of applicability.
\end{abstract}

Keywords Alpine skiing $\cdot$ Modelling $\cdot$ Balance/stability $\cdot$ Performance

\section{Introduction}

The interaction between skis and snow is one of the most important aspects of skiing. For a classical glider, the interaction with the surface it glides reduces to the normal to the surface reaction force $N$ and the Coulomb friction force $\boldsymbol{F}_{\mathrm{f}}=-\mu N \hat{\boldsymbol{m}}$, where $\mu$ is the kinetic coefficient of friction, and $\hat{\boldsymbol{m}}$ is the unit vector in the direction of relative motion. The physics of friction between snow and ski is interesting and important (e.g. [1-3]). However, it has been long recognised that there must be more to the snow-ski interaction. Indeed, neither of the above two forces can change the direction of motion and hence explain how skiers manage to execute ski turns.

It is also well known to every skier that during turns their skis are not flat on the snow but set on their inside edges instead. Furthermore, in so-called skidded (or steered) turns, edged skis slip sideways and, in the process, remove a layer of snow with their edges. In this regard, a ski works like a cutting tool in industrial machining of various materials. Understandably, the mechanics of industrial machining has

This article is a part of Topical Collection in Sports Engineering on Winter Sports, edited by Dr. Aimee Mears, Dr. David Pearsall, Dr. Irving Scher and Dr. Carolyn Steele.

Serguei S. Komissarov

s.s.komissarov@leeds.ac.uk

1 School of Mathematics, The University of Leeds, Leeds LS2 9JT, UK been a subject of intensive research, both experimental and theoretical [4-9, to name few]. Much less attention has been attracted to the machining of ice and snow, which has no industrial applications. A laboratory study of ice machining was carried out by [10], and machining of compacted snow was studied in [11-13]. They obtained rather complicated empirical expressions for the forces emerging in the process. Although these complex expressions are not particularly convenient for theoretical analysis, they could still be used in numerical integration of the equations of motion. [14] proposed to apply the theory of metal machining instead. Most importantly, these studies demonstrated the presence of the turning force orthogonal to the direction of motion.

Recently, [15] presented a simple approximate analytical theory of snow machining and compared it against the data of former laboratory experiments. The results were encouraging and this approach is developed further by applying the theory to the most basic manoeuvres of alpine skiing, side-slipping down fall line, hockey stop, and traversing. Our aim is to check if the results agree with the practice of alpine skiing and to lay foundation for future verification of the theory in experiments on real ski slopes. The simplicity of these manoeuvres makes setting up of such experiments quite straightforward. 


\section{Methods and materials}

Following [15], it is assumed here that the snow reaction force $\boldsymbol{F}_{\mathrm{r}}$ is normal to the ski base. Since the ski is normally put on its edge, one can split $\boldsymbol{F}_{\mathrm{r}}$ into components normal and tangent to the surface of the ski slope,

$\boldsymbol{F}_{\mathrm{r}}=\boldsymbol{N}+\boldsymbol{F}_{\mathrm{c}}$.

The normal component $\boldsymbol{N}=N \hat{\boldsymbol{k}}(N>0)$, where $\hat{\boldsymbol{k}}$ is a outward unit normal to the slope surface, is known in the theory of machining as the feed force. The tangential component, $\boldsymbol{F}_{\mathrm{c}}$ is known as the cutting force. If $\hat{\boldsymbol{n}}_{\mathrm{s}}$ is a unit vector tangent to slope, normal to the ski edge, and pointing to the side opposite to the direction of motion, then $\boldsymbol{F}_{\mathrm{c}}=F_{\mathrm{c}} \hat{\boldsymbol{n}}_{\mathrm{s}}$ $\left(F_{\mathrm{c}}>0\right)$. Obviously,

$F_{\mathrm{c}}=N \tan \Psi$,

where $\Psi$ is the edge angle, the angle between the ski base and the slope surface. If $\hat{\boldsymbol{s}}$ is the unit vector aligned with the ski, and $\hat{\boldsymbol{m}}$ is the unit vector in the direction of ski motion relative to the snow, then

$\hat{\boldsymbol{n}}_{\mathrm{s}}=\frac{(\hat{\boldsymbol{m}} \times \hat{\boldsymbol{s}}) \times \hat{\boldsymbol{s}}}{|\hat{\boldsymbol{m}} \times \hat{\boldsymbol{s}}|}$.

In the tangent plane of the slope, $\boldsymbol{F}_{\mathrm{c}}$ splits into the braking force $\boldsymbol{F}_{\mathrm{b}}=-F_{\mathrm{b}} \hat{\boldsymbol{m}}\left(F_{\mathrm{b}}>0\right)$ and the turning force $\boldsymbol{F}_{\mathrm{t}}=F_{\mathrm{t}} \hat{\boldsymbol{n}}_{\mathrm{m}}\left(F_{\mathrm{t}}>0\right)$, where

$\hat{\boldsymbol{n}}_{\mathrm{m}}=\frac{(\hat{\boldsymbol{m}} \times \hat{\boldsymbol{s}}) \times \hat{\boldsymbol{m}}}{|\hat{\boldsymbol{m}} \times \hat{\boldsymbol{s}}|}$.

It is easy to see that

$F_{\mathrm{b}}=F_{\mathrm{c}} \sin \gamma$ and $F_{\mathrm{t}}=F_{\mathrm{c}} \cos \gamma$,

where $0 \leq|\gamma|<90^{\circ}$ is the angle of attack, the angle between the ski edge and its direction of motion. Thus, the cutting force contributes mostly to braking at large angles of attack and mostly to turning at small angles of attack.

In this theory, skis are modelled as straight planks and hence their non-trivial shape and ability to bend and twist are completely ignored. These complicating factors will have to be taken into account in more advanced models.

The other two key forces acting on the skier are the gravity force

$\boldsymbol{F}_{\mathrm{g}}=M \boldsymbol{g}$,

where $M$ is the skier mass and $\boldsymbol{g}$ is the gravitational acceleration, and the aerodynamic drag force

$\boldsymbol{F}_{\mathrm{d}}=-\frac{C_{d} A \rho}{2} v^{2} \hat{\boldsymbol{m}}$ where $C_{d}$ is the drag coefficient, $A$ is the cross-section area of the skier normal to the direction of motion, $\rho$ is the mass density of the air, and $v$ is the skier speed relative to the air [2]. Hence, the motion of the skier centre of mass (CM) is governed by the equation

$M a=F_{\mathrm{g}}+\boldsymbol{F}_{\mathrm{r}}+\boldsymbol{F}_{\mathrm{d}}$,

where $\boldsymbol{a}$ is the CM acceleration. The strong dependence of $\boldsymbol{F}_{\mathrm{d}}$ on the speed ensures that at sufficiently low speeds it can be safely ignored.

\section{Results}

\subsection{Side-slipping down the fall line}

As a first application to skiing, let us consider one of its most basic manoeuvres, side-slipping down the fall line (the line of steepest descent down the ski slope). Since this manoeuvre is executed with skis directed perpendicular to the fall line, it corresponds to the case of orthogonal machining (see Fig. 1). Normally, this manoeuvre is performed at low speed, which allows to ignore the contribution of aerodynamic drag in Eq. (6).

Let us consider a ski slope with the slope angle $\alpha$, the angle between the slope plane and the horizontal plane. Using Cartesian coordinates with the basis vectors $\hat{\boldsymbol{k}}$ (outward normal to the surface of the ski slope), $\hat{i}$ (parallel and down to the fall line), and $\hat{j}$ (perpendicular to the other two), one can write $\boldsymbol{a}=a \hat{\boldsymbol{i}}, \boldsymbol{g}=g \sin \alpha \hat{\boldsymbol{i}}-g \cos \alpha \hat{\boldsymbol{k}}$, $\boldsymbol{N}=N \hat{\boldsymbol{k}}$, and $\boldsymbol{F}_{\mathrm{r}}=-N \tan \boldsymbol{\Psi \boldsymbol { i }}+N \hat{\boldsymbol{k}}$. Hence the component of Eq. (6) along $\hat{\boldsymbol{k}}$ reads

$N=M g \cos \alpha$.

This is the usual condition of force balance perpendicular to the slope. The component of Eq. (6) along $\hat{i}$ determines the magnitude of the CM acceleration,

$a=g \cos \alpha(\tan \alpha-\tan \Psi)$.

This result tells us that the edge angle allows control of sideslipping. Namely,

$\Psi<\alpha$ for acceleration ;

$\Psi=\alpha$ for constant speed ;

$\Psi>\alpha$ for deceleration .

This conclusion is fully consistent with skiing practice. Moreover, the condition $\Psi=\alpha$ also agrees with the fact that, during side-slipping at constant speed, the skier's shanks are aligned with the vertical direction (e.g. Fig. 1). 

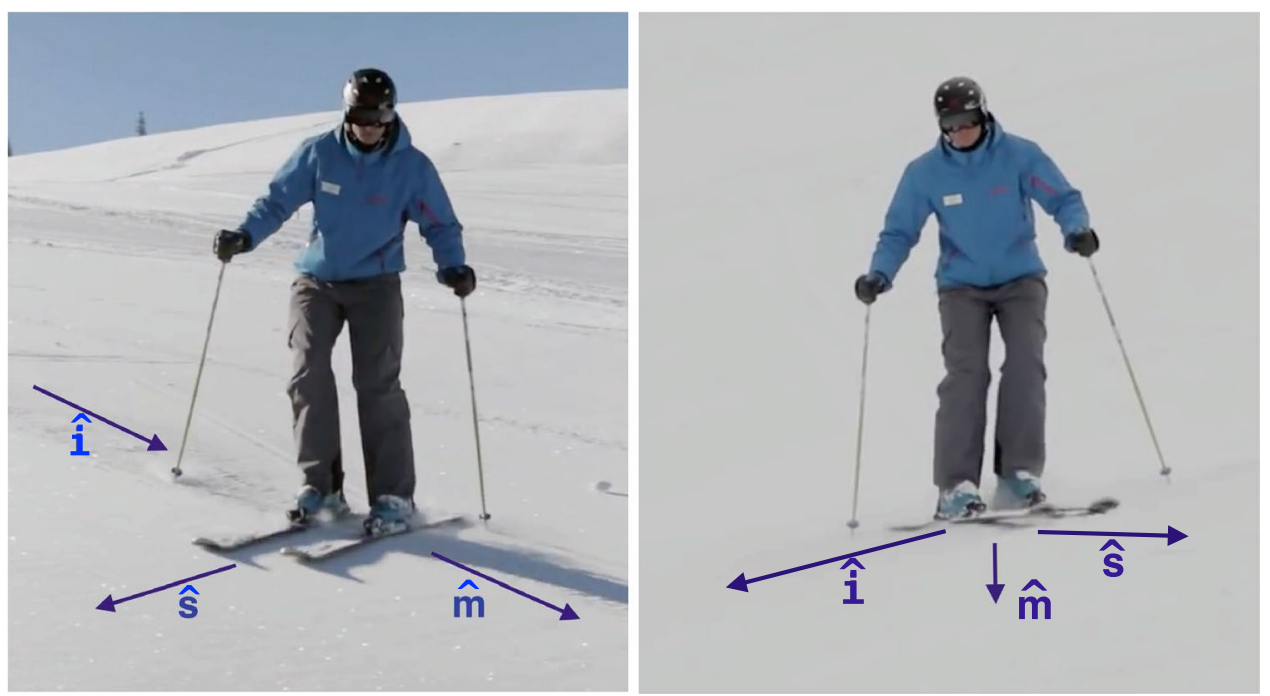

Fig. 1 Left panel: a skier side-slipping down the fall line. Right panel: a skier traversing a ski slope at fixed angle of attack. These are frames from the video "Alltracks Ski Tips. Skiing with flow" (https:// www.youtube.com/watch? $\mathrm{v}=$ NoQsLdRD7nE, frame times 1:48 and 1:56). $\hat{\boldsymbol{m}}$ is the direction of motion, $\hat{\boldsymbol{i}}$ is the direction of the fall line, and $\hat{s}$ is the direction of the ski edge; these are drawn based on the

\subsection{Hockey stop}

In the "hockey stop" manoeuvre, skiers (skaters) quickly pivot their skis (skates) to the angle of attack $\gamma \approx 90^{\circ}$ and push them ahead of their CM in the direction of motion. The theory of snow machining supports this action as the most effective way of braking. Indeed, pushing skis forward results in a higher edge angle and hence a higher snow cutting force (see Eq. 2), whereas for a given cutting force, the braking force is maximised at $\gamma=90^{\circ}$ (see Eq. 5). One can use Eq. (8) to obtain crude estimates of the braking time and distance to a full stop

$T_{\mathrm{br}}=V_{0} / a \quad$ and $\quad L_{\mathrm{br}}=\frac{V_{0}^{2}}{2 a}$

respectively, where $V_{0}$ is the initial speed. For $\alpha=15^{\circ}$ and $V_{0}=10 \mathrm{~m} / \mathrm{s}$, Eq. (8) predicts $a=7.1 \mathrm{~m} / \mathrm{s}^{2}, T_{\mathrm{br}}=1.4 \mathrm{~s}$, and $L_{\mathrm{br}}=7.0 \mathrm{~m}$ if $\Psi=45^{\circ}$ and $a=14 \mathrm{~m} / \mathrm{s}^{2}, T_{\mathrm{br}}=0.7 \mathrm{~s}$, and $L_{\mathrm{br}}=3.5 \mathrm{~m}$ if $\Psi=60^{\circ}$. These values seem quite realistic, though the author could not find any experimental study of the manoeuvre required for quantitative comparison.

\subsection{Skidded traversing}

The second basic skiing manoeuvre considered here, is the straight line skidded traverse (diagonal side-slipping). For a motion along straight line, the total force acting on the body "naked-eye" analysis of the video. (The images are used with permission from Alltracks Academy, West Tytherley, Hampshire, SP5 1LX, UK). The reader may also find useful the following two videos: https://www.youtube.com/watch? $\mathrm{v}=\mathrm{fS} 1 \mathrm{BS}$ apSyos and https://vimeo. com/user2580425 (the video titled Ski Improvement: Separation through the arc around the play time 1:00)

must be aligned with the velocity vector. If the skier was experiencing only the gravity force, aerodynamic drag, and Coulomb friction, such motion would be impossible because the drag and friction forces are aligned with the velocity, but the gravity force is not and pulls the skier towards the fall line.

In contrast, the snow cutting force has a component (the turning force) perpendicular to the velocity vector. If this component can balance the perpendicular to the velocity component of the gravity force, then straight line traversing is possible. Here, we explore this possibility.

Now $\boldsymbol{a}=a \hat{\boldsymbol{m}}$ and $\boldsymbol{F}_{\mathrm{r}}=N \tan \Psi \hat{\boldsymbol{n}}_{\mathrm{s}}+N \hat{\boldsymbol{k}}$. Let us denote the angle between the velocity vector and the fall line as $\beta$ (the angle of traverse) and agree that the angle of attack $-90^{\circ} \leq \gamma \leq 90^{\circ}$ is positive in the rotational direction away from the fall-line (see Fig. 2). Hence $\hat{\boldsymbol{m}}=(\cos \beta, \sin \beta, 0)$ and $\boldsymbol{n}_{\mathrm{s}}=\operatorname{sign}(\gamma)(-\sin (\gamma+\beta), \cos (\gamma+\beta), 0)$.

In the direction of $\hat{k}$, the equation of motion (6) still yields Eq. (7). However, in the slope plane

$(a / g) \hat{\boldsymbol{m}}=\sin \alpha \hat{\boldsymbol{i}}+\cos \alpha \tan \Psi \hat{\boldsymbol{n}}_{\mathrm{s}}-f_{\mathrm{d}} \hat{\boldsymbol{m}}$,

where $f_{\mathrm{d}}=F_{\mathrm{d}} / M g=C_{\mathrm{d}} A \rho v^{2} / 2 M g$ is the drag force in the units of $M g$. In the direction normal to $\hat{\boldsymbol{m}}$ (and hence parallel to the vector $\left.\hat{\boldsymbol{n}}_{\mathrm{m}}=(-\sin \beta, \cos \beta, 0)\right)$, this equation yields

$\tan \alpha \sin \beta=\operatorname{sign}(\gamma) \tan \Psi \cos \gamma$.

Since $0^{\circ}<\alpha, \beta, \Psi<90^{\circ}$, this equation can be satisfied only if $\gamma>0$. This agrees with the fact that, in traversing, skis are pivoted away from the fall line (see Fig. 2). Conversely, 


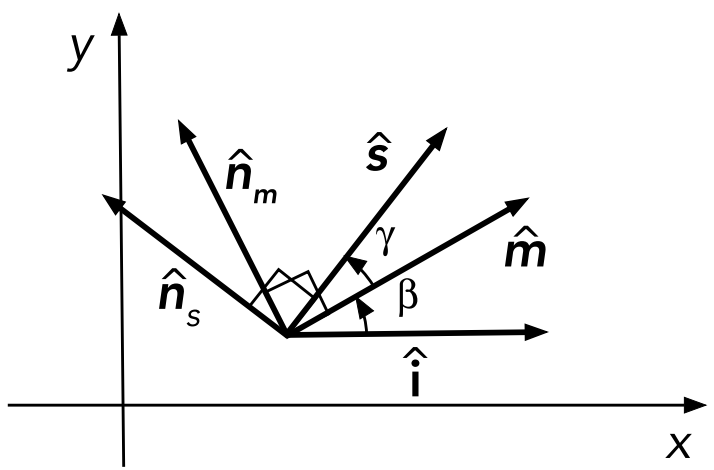

Fig. 2 Key unit vectors and angles in the plane of the tangent plane of ski slope which emerge in the problem of traverse. $\hat{\boldsymbol{i}}$ is the direction of fall line, $\hat{\boldsymbol{m}}$ is the direction of motion, $\hat{\boldsymbol{s}}$ is the direction of the ski edge, vector $\hat{\boldsymbol{n}}_{\mathrm{m}}$ is normal to $\hat{\boldsymbol{m}}$ and $\hat{\boldsymbol{n}}_{\mathrm{s}}$ is normal to $\hat{\boldsymbol{s}}$. $\boldsymbol{\beta}$ is the angle of traverse and $\gamma$ is the angle of attack

if skis are pivoted towards the fall line $(\gamma<0)$, the trajectory has to be curved. Indeed, this is how skis are pivoted in skidded turns.

For the motion along the fall line $(\beta=0)$, Eq. (11) implies $\gamma=90^{\circ}$. This justifies the assumption $\gamma=90^{\circ}$ made, on the basis of observations, in our analysis of side-slipping down the fall line.

In the direction of $\hat{\boldsymbol{m}}$, Eq. (10) yields

$a / g=\sin \alpha \cos \beta-|\sin \gamma| \tan \Psi \cos \alpha-f_{\mathrm{d}}$.

Normally, skidded traverse is performed at low speed, which allows us to ignore the drag force $\left(f_{\mathrm{d}} \rightarrow 0\right)$. Moreover, once the speed growth has saturated, one can put $a=0$. Under these conditions, Eqs. (11) and (12) lead to

$\tan \Psi=\tan \alpha \quad$ and $\quad \beta+\gamma=90^{\circ}$.

Hence, like in the case of side-slipping down to the fall line, skidded traversing also involves vertical position of skier's shanks and skis placed perpendicular to the fall line. This explains why skier's postures in orthogonal and traverse side slipping look very much the same (compare the images in Fig. 1.)

\subsection{Rotational balance of skiers}

The above results imply that changing the edge angle is the main way of controlling the speed of traverse. In industrial machining, where the tool is firmly held in its place by fixing devices, one can set almost any edge angle for a cutting tool. In skiing, the range is more limited because skiers have to find a balanced position over their skis, or they will crash. For exact balance, the torque acting on the skier has to vanish (small deviations from this condition can be utilised for control over skier's orientation.)

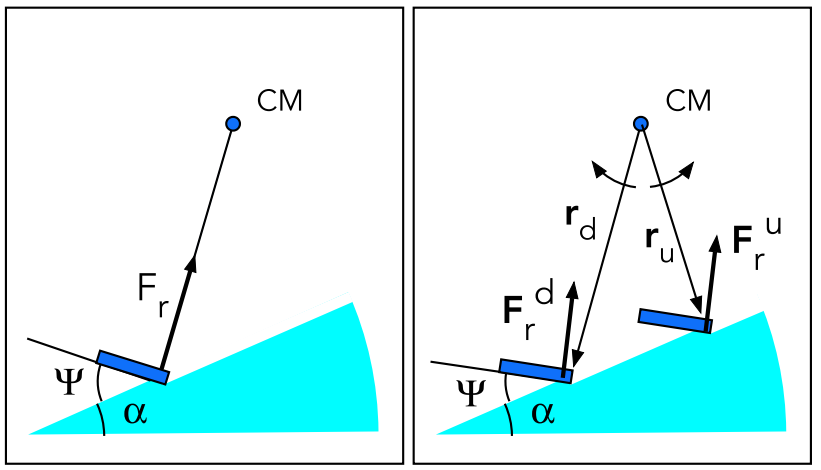

Fig. 3 Left panel: rotational balance in the case of side-slipping on one ski. The snow reaction force $\boldsymbol{F}_{\mathrm{r}}$ points directly toward the skier centre of mass $(\mathrm{CM})$ and the torque due to the snow reaction force about the $\mathrm{CM}$ vanishes. Right panel: rotational balance in the case of side-slipping on both skis. The CM is located between the lines normal to the ski bases and originating from cutting edge. The torques due to the snow reaction forces originating at the downhill and uphill skis point in the opposite directions and balance each other

Since both the gravity and the inertial forces associated with skier's acceleration can be treated as applied at skier's $\mathrm{CM}$, the condition of rotational balance can be formulated as vanishing of the torque relative to the $\mathrm{CM}$ due to the snow reaction force and the aerodynamic drag. Here the focus is on the case where the torque due to the drag can be neglected ${ }^{1}$. This is reasonable because the aerodynamic drag force grows like $v^{2}$, where $v$ is the skier speed relative to air, and becomes small at sufficiently low speeds. Moreover, the application area of the drag force is spread around the CM and hence the effective lever must be significantly shorter compared to that of the snow reaction force.

When skier is balancing on one ski, the condition of rotational balance reads

$\boldsymbol{r} \times \boldsymbol{F}_{\mathrm{r}}=0$,

where $\boldsymbol{r}$ is the position vector of the cutting edge of this ski relative to the skier's CM, and $\boldsymbol{F}_{\mathrm{r}}$ is the snow reaction force applied to this ski. Thus, $\boldsymbol{F}_{\mathrm{r}}$ must point directly at the CM (see the left panel of Fig. 3). Maintaining this alignment involves continuous correction of the edge angle via changing the knee angulation and requires advanced skills.

Normally, skiers balance on both skis. In this case, the rotational balance reads

$\boldsymbol{r}^{d} \times \boldsymbol{F}_{\mathrm{r}}^{d}+\boldsymbol{r}^{u} \times \boldsymbol{F}_{\mathrm{r}}^{u}=0$

where suffixes ' $u$ ' and 'd' refer to the uphill and downhill skis, respectively. For simplicity, let us assume that both the

\footnotetext{
${ }^{1}$ Effectively, this reduces the rotational balance to the plane normal to the ski edge.
} 

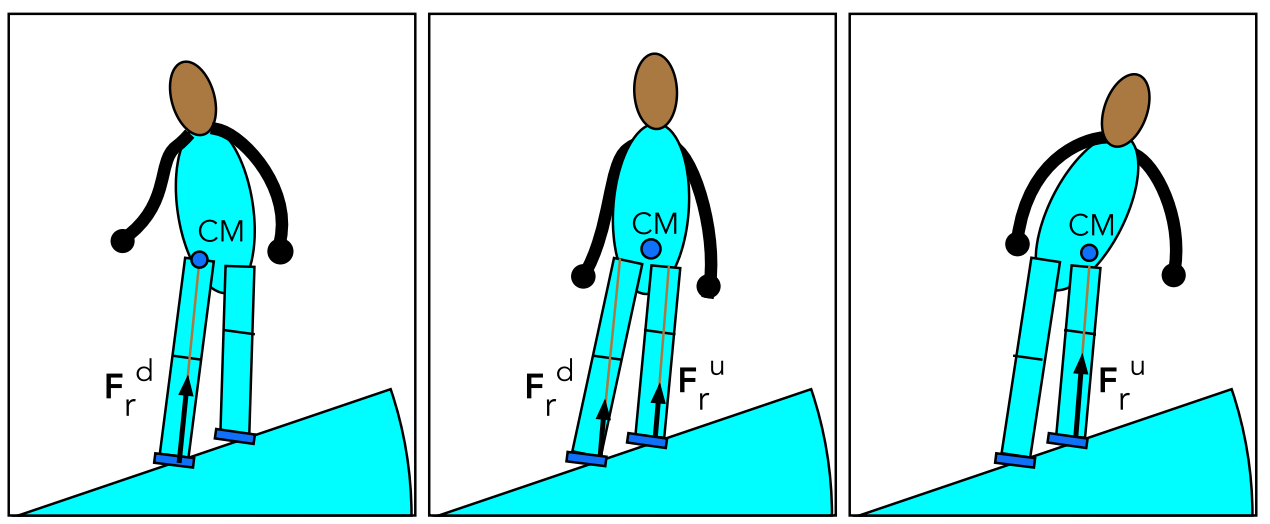

Fig. 4 Posturing of rotationally balanced skier depending on the load distribution between skis. Left panel: the skier keeps the entire load on the downhill ski and the CM is shifted downhill toward the continuation of the snow reaction force vector originating at the cutting

skis have exactly the same edge angle and hence $\boldsymbol{F}_{\mathrm{r}}^{d} \| \boldsymbol{F}_{\mathrm{r}}^{u}$. Hence, one can write $\boldsymbol{F}_{\mathrm{r}}^{d}=\kappa \boldsymbol{F}_{\mathrm{r}}^{t}$ and $\boldsymbol{F}_{\mathrm{r}}^{u}=(1-\kappa) \boldsymbol{F}_{\mathrm{r}}^{t}$, where $\boldsymbol{F}_{\mathrm{r}}^{t}=\boldsymbol{F}_{\mathrm{r}}^{u}+\boldsymbol{F}_{\mathrm{r}}^{d}$ is the total snow reaction force and $0 \leq \kappa \leq 1$ is the loading parameter. In this case, the condition of rotational balance reduces to

$\left(\boldsymbol{r}^{u}+\kappa\left(\boldsymbol{r}^{d}-\boldsymbol{r}^{u}\right)\right) \times \boldsymbol{F}_{\mathrm{r}}^{t}=0$.

Obviously, the vector $\boldsymbol{r}_{\mathrm{b}}=\boldsymbol{r}^{u}+\kappa\left(\boldsymbol{r}^{d}-\boldsymbol{r}^{u}\right)$ connects the CM to a point on the slope located between the cutting edges of the skis. For the rotational balance, this vector must be aligned with $\boldsymbol{F}_{\mathrm{r}}^{t}$. Such a vector exists provided the CM resides between the cutting edges, when projected on the plane of the ski base (the plane normal to $\boldsymbol{F}_{\mathrm{r}}^{t}$, see the right panel of Fig. 3). Skiers can maintain this balance via adjusting the load distribution, which does not require any more skill than standing on two feet. Moreover, when the skier is in translational balance as well, and hence $\boldsymbol{F}_{\mathrm{r}}^{t}=-m \boldsymbol{g}$, their $\mathrm{CM}$ must be located above their base of support. This is exactly the same rotational balance condition as for standing on a floor.

If only the downhill ski is loaded, then $\kappa=1$ and $\boldsymbol{r}_{\mathrm{m}}=\boldsymbol{r}^{d}$. Hence, the CM is shifted to the extreme downhill position relative to the skis. Similarly, when only the uphill ski is loaded, $\kappa=0, \boldsymbol{r}_{\mathrm{m}}=\boldsymbol{r}^{u}$, and the CM is shifted to the extreme uphill position. Such shifts are normally achieved via angulating of skier's body (see Fig. 4).

\section{Discussion}

Straight line traversing has been studied experimentally by [16], with the aim of determining the coefficient of kinetic friction and the aerodynamic drag area in alpine skiing. They set a $30 \mathrm{~m}$ long corridor at traverse angle $\beta=52^{\circ}$ on a slope edge of this ski. Right panel: the skier keeps the entire load on the uphill ski and the CM is shifted uphill. Middle panel: the skier distributes the load evenly between the downhill and uphill skis

with inclination $\alpha=23^{\circ 2}$. The participating skier was asked to avoid side-slipping. With zero sidecut skis, this should be possible. On shaped skis, however, this is problematic because shaped skis tend to carve an arc instead of a straight line when run on the edge. Indeed, [16] reported that the skier could not perform the task exactly and that some sideslipping did occur, resulting in increased snow resistance. Although this experiment was not focused on side-slipping and hence is not well-suited for our purposes, we could not find anything better in the literature. Here, the parameters of side-slipping are estimated assuming it was a permanent feature of the runs.

It is easy to see that Eq. (12) can be written as

$a / g=\sin \alpha \cos \beta-\mu_{\mathrm{eff}} \cos \alpha-f_{\mathrm{d}}$,

where

$\mu_{\text {eff }}=\tan \Psi \sin \gamma$.

This equation is identical to Eq. (22) in [16] in all respects apart from the coefficient of Coulomb friction $\mu$ being replaced by $\mu_{\text {eff }}$. This suggests that the values of $\mu$ reported in [16] actually measure $\mu_{\text {eff }}$. Solving Eq. (18) together with the straight-traverse condition (11) for $\gamma$ and $\Psi$ one obtains

$\tan \gamma=\frac{\mu_{\text {eff }}}{\tan \alpha \sin \beta}$

and

\footnotetext{
2 The data used in our calculations were communicated to us by the authors of [16], who had realised that their paper had few factual
} inaccuracies. 
Table 1 Parameters of straight traverse runs studied in [16]

\begin{tabular}{lllllll}
\hline run & $v_{\mathrm{s}}$ & $v_{\mathrm{f}}$ & $\mu^{*}$ & $\mu_{\text {eff }}$ & $\gamma\left({ }^{\circ}\right)$ & $\Psi\left({ }^{\circ}\right)$ \\
\hline 1 & 0.6 & 10.6 & 0.064 & 0.051 & 8.6 & 19 \\
2 & 11.0 & 13.4 & 0.128 & 0.067 & 11 & 19 \\
3 & 14.7 & 16.6 & 0.153 & 0.053 & 9.0 & 19 \\
\hline
\end{tabular}

The initial speed $v_{\mathrm{s}}$, the final speed $v_{\mathrm{f}}$, and the "friction" coefficient $\mu^{*}$ are the parameters given in [16]. $\mu_{\mathrm{eff}}$ is the effective coefficient of kinetic friction as defined by Eq. 18 and estimated using Eq. 21. $\gamma$ is the attack angle calculated using Eq. 19, and $\Psi$ is the ski edge angle calculated using Eq. 20 $\tan \Psi=\frac{\mu_{\text {eff }}}{\sin \gamma}$.

Hence, one can convert the measured values of $\mu_{\text {eff }}$ into the geometrical parameters of side-slipping and quickly check if they are reasonable or not.

Unfortunately, the values of the friction coefficient reported in [16] are calculated under the assumption of negligible aerodynamic drag. Denoting them as $\mu^{*}$, one has

$\mu^{*}=\mu_{\mathrm{eff}}+\frac{f_{\mathrm{d}}}{\cos \alpha}$.

To extract $\mu_{\text {eff }}$ from the data, one needs to estimate the aerodynamic drag force $f_{\mathrm{d}}$. The values of $\mu_{\text {eff }}$ shown in Table 1 are calculated assuming the skier weight $M=88 \mathrm{~kg}$, the reasonable for an upright skier $C_{\mathrm{d}} A=0.6 \mathrm{~m}^{2}$, the air density at one kilometre above the sea level $\rho=1.08 \mathrm{~kg} \mathrm{~m}^{-3}$, and the mean value of the skier speed. One can see that the braking due to the aerodynamic drag is comparable to the braking associated with the snow reaction force. This is a consequence of the quite high skier speed in these runs.

The estimated value of $\mu_{\text {eff }}$ are significantly lower than $\mu^{*}$ but still well above the value of $\mu=0.0085$ for the proper snow friction measured by [16] in the experiment involving fall-line gliding. This suggests that the snow cutting force was dominant over the friction in the traversing runs.

Interestingly, our calculations yield more or less the same attack and edge angles for all the three runs (see Table 1). This suggests that the skier executed all runs in the more or less the same manner. None of the angles were measured in [16]. This is rather unfortunate because such measurements are needed to test our model. Using modern technology this can be done quite accurately [17, e.g.].

Although this paper deals only with motions along straight line, side-slipping is an integral part of many skiing turns. Some types of skiing turns, e.g. the wedge turn or the classic parallel turn, are skidded from start to finish. Modelling of such turns based on the same model of snow machining will be presented elsewhere.

Other types of turns combine skidding with carving. They start just like skidded turns with pivoting of skis and developing a non-vanishing angle of attack, but during the turn the angle of attack reduces and the turn finishes without side-slipping [17, e.g.]. The theory of machining offers a plausible explanation of the transition. When skis are not rotated during skidding, the braking component of the cutting force reduces the velocity component normal to the skis and hence the angle of attack. Naturally, $\gamma \rightarrow 0$ signifies transition to carving. At this point, the braking component of the cutting force vanishes, whereas its turning component $F_{\mathrm{t}} \rightarrow N \tan \Phi$, which is the same as the turning force in balanced carving $[18,19]$. This can be the reason for the seamless transition between skidding and carving phases of a hybrid turn, when executed by a proficient skier. Hybrid turns are among the most advanced tools in the arsenal of expert skiers and ski racers and require rather advanced understanding of skiing mechanics for successful modelling.

\section{Conclusion}

In this paper, a simple model of ski-snow interaction based on the theory of machining of materials is used to describe the most basic manoeuvres of alpine skiing involving skidding along a straight line: side-slipping down the fall line, hockey stop, and traversing. The results are found to be consistent with the common practice of alpine skiing, e.g. with the use of edge angle to control side-slipping, with the orthogonal to the fall line orientation of skis and vertical orientation of skier's shanks during constant-speed traverse, and with the efficiency of a hockey stop. The model also explains the abnormally high values of the kinetic friction coefficient found in previous models based on Coulomb friction. On the other hand, the quantitative accuracy of the model remains unknown due to the current lack of relevant experimental data. We hope that our theory will stimulate experimental research into side-slipping and this important question will soon be answered.

Acknowledgements We are grateful to anonymous reviewers for encouraging and constructive comments that helped to improve this paper. We are particularly thankful to the referee who was also one of the authors of [16] for reviewing their records and providing us with further details of their investigation. 


\section{Declarations}

Conflict of interest The authors declare that they have no conflict of interest.

Open Access This article is licensed under a Creative Commons Attribution 4.0 International License, which permits use, sharing, adaptation, distribution and reproduction in any medium or format, as long as you give appropriate credit to the original author(s) and the source, provide a link to the Creative Commons licence, and indicate if changes were made. The images or other third party material in this article are included in the article's Creative Commons licence, unless indicated otherwise in a credit line to the material. If material is not included in the article's Creative Commons licence and your intended use is not permitted by statutory regulation or exceeds the permitted use, you will need to obtain permission directly from the copyright holder. To view a copy of this licence, visit http://creativecommons.org/licenses/by/4.0/.

\section{References}

1. Colbeck SC (1992) A review of the processes that control snow friction. In: Technical report, Cold Regions Research and Engineering Laboratory (CRREL), Hanover, NH. https://apps.dtic.mil/ sti/citations/ADA252362. Accessed 1 June 2021

2. Lind D, Sanders SP (1996) The physics of skiing: skiing at the triple point. Springer, New York

3. Nachbauer W, Kaps P, Hasler M, Mössner M (2016) Friction between ski and snow. In Braghin F, Cheli F, Maldifassi S, Melzi S (eds) The engineering approach to winter sports, 2016, Springer, New York, p 33

4. Merchant ME (1945a) Mechanics of the metal cutting process. I. Orthogonal cutting and a type 2 chip. J Appl Phys 16:267-275

5. Merchant ME (1945b) Mechanics of the metal cutting process. ii. Plasticity conditions in orthogonal cutting. J Appl Phys 16:318-324

6. Lin GCI, Oxley PLB (1972) Mechanics of oblique machining: predicting chip geometry and cutting forces from work material properties and cutting conditions. Proc Inst Mech Eng 186:813-820

7. Stevenson R (1998) The measurement of parasitic forces in orthogonal cutting. Int J Mach Tools Manuf 38(1):113-130

8. Wyen C-F, Wegener KK (2010) Influence of cutting edge radius on cutting forces in machining titanium. CIRP Ann 59(1):93-96

9. Guo YB, Chou YK (2004) The determination of ploughing force and its influence on material properties in metal cutting. J Mater Process Technol 148(3):368-375

10. Lieu DK, Mote CD (1984) Experiments in the machining of ice at negative rake angles. J Glaciol 30:77-81

11. Tada N, Hirano Y (1999) Simulation of a turning ski using ice cutting data. Sports Eng 2(1):55-64

12. Tada N, Hirano Y (2002) In search of the mechanics of a turning alpine ski using snow cutting force measurements. Sports Eng 5:15-22

13. Tada N, Kobayashi T (2005) Measurement of snow cutting forces for analysis and design of a snow ski. J Ski Sci 3:11-21

14. Brown CA (2009) Modeling edge-snow interactions using machining theory. In: Müller E, Lindinger S, Stöggl T (eds) Science and skiing IV. Meyer \& Meyer Sport, Maidenhead, pp 175-182

15. Komissarov SS (2021) Mechanics of side-slipping in alpine skiing: theory of machining snow and ice. Sports Eng 24:4

16. Kaps P, Nachbauer W, Mössner M (1996) Determination of kinetic friction and drag area in alpine skiing. In: Mote C, Johnson R, Hauser W, Schaff P (eds) Skiing trauma and safety: tenth volume. ASTM International, West Conshohocken, pp 165-177

17. Reid RC (2010) A kinematic and kinetic study of alpine skiing technique in slalom. Phd dissertation, Norwegian School of Sport Sciences. http://hdl.handle.net/11250/171325. Accessed 1 June 2021

18. Howe J (1983) Skiing mechanics. Poudre Press, Laporte

19. Komissarov SS (2020) Balanced carving turns in alpine skiing. Sports Biomech. https://doi.org/10.1080/14763141.2020.1795236

Publisher's Note Springer Nature remains neutral with regard to jurisdictional claims in published maps and institutional affiliations. 